\title{
Impact of the A48 Collimator on the Tevatron BØ Dipoles
}

\author{
L.Y. Nicolas, N.V. Mokhov \\ Fermi National Accelerator Laboratory \\ P.O. Box 500, Batavia, Illinois 60510, USA
}

June 17, 2003

\begin{abstract}
Energy deposition in the BØ dipoles downstream of a new A48 collimator to be installed in the Tevatron this summer is calculated with the MARS14 code to evaluate the dipole's quench stability at an abort kicker prefire.
\end{abstract}




\section{Introduction}

To protect the CDF detector components in an event of an abort kicker prefire (AKP) in the Tevatron, a new collimator is to be installed at the A48 location during the summer 2003 shutdown [1, 2]. Detailed calculations have shown that this $0.5-\mathrm{m}$ long "single L-shape" steel collimator will intercept a bunch of protons when such an incident occurs, providing reliable protection of the CDF main detector at an AKP. It will also mitigate the backgrounds induced by elastic beam-gas interactions upstream of $\mathrm{B} \varnothing[3,4]$. Although the Roman Pot detectors downstream of the A48 collimator will see an increased background, the amount of radiation they will receive either resulting from beam halo interactions in the collimator or during an AKP will not damage their sensitive parts.

Secondaries resulting from beam halo interactions with the A48 collimator do not noticeably affect the downstream dipoles. The case of an AKP is quite different. As opposed to halo hits in the "single-L shape" unit (around $10^{5} \mathrm{p} / \mathrm{s}$ ), a bunch lost on A48 during an AKP represents more than $2 \times 10^{11}$ protons "instantaneously" interacting with the collimator material. Although the collimator protects the downstream superconducting (SC) dipoles against a damage in such an event, secondaries generated in A48 create a significant radiation load on the dipoles which will most likely result in a quench of the first one. This effect is studied in detail in this note.

\section{Modeling}

Tracking of a misbehaved proton beam is performed with the STRUCT code [5] for the cases of Tevatron proton and antiproton abort kicker prefires. Fig. 1 (left) is a scattered plot of protons on A48 at an AKP. The showers generated in the collimator and downstream components at such an event and corresponding energy deposition are calculated with the MARS14 code [6]. All necessary details of the geometry, materials and magnetic fields are included in the model. Results of the simulations are normalized per $2.77 \times 10^{11}$ protons in a bunch at A48. Fig. 1 (right) shows a 2D snapshot of the region including the Tevatron beam pipe, a SC dipole downstream of the A48 collimator, and a secondary particle track sample at an AKP.
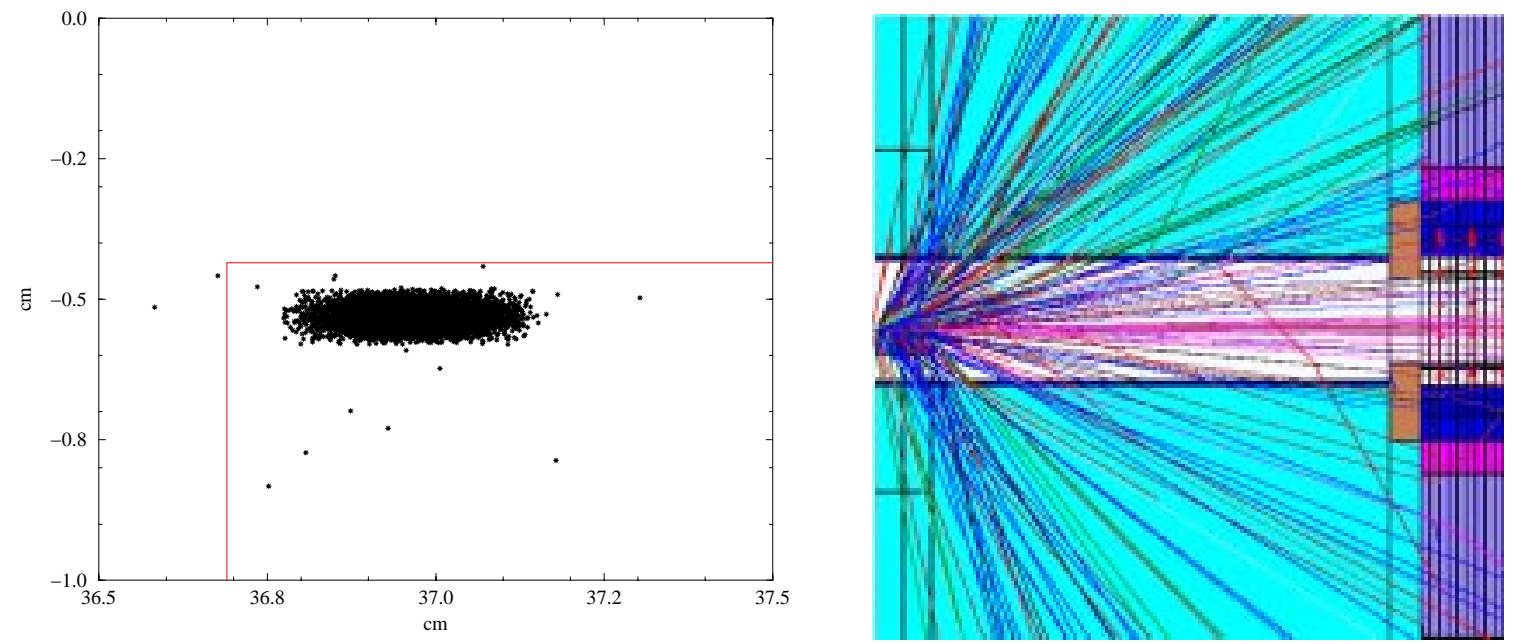

Figure 1: Proton hit distribution on a A48 jaw at an AKP (left) and a sample of secondary particle tracks in the collimator-mask-dipole system (right). 


\section{Baseline}

First, the baseline configuration with the proposed $0.5-\mathrm{m}$ long steel collimator followed by the SC dipole string was simulated. Fig. 2 (left) gives some details of the dipole model in calculations, while Fig. 2 (right) shows energy deposition distribution at shower maximum which takes place at about $65 \mathrm{~cm}$ from the non-IP end of the first dipole downstream of the A48 collimator. It turns out that energy deposition decays rapidly along the dipole length, being substantially lower in the second and third dipoles (those closer to the IP). Therefore the analysis below is limited to the first meter of the first dipole. Fig. 3 shows longitudinal energy deposition profile, separately for the right/left and inner/outer SC coils. The peak energy deposition reaches $17 \mathrm{~mJ} / \mathrm{g}$ in the inner coil, that is 34 times higher than the quench limit of $0.5 \mathrm{~mJ} / \mathrm{g}$. It is clear that to reduce maximum energy deposition, the A48 collimator length needs to be increased, and possibilities for heavier materials and additional masks should be explored.
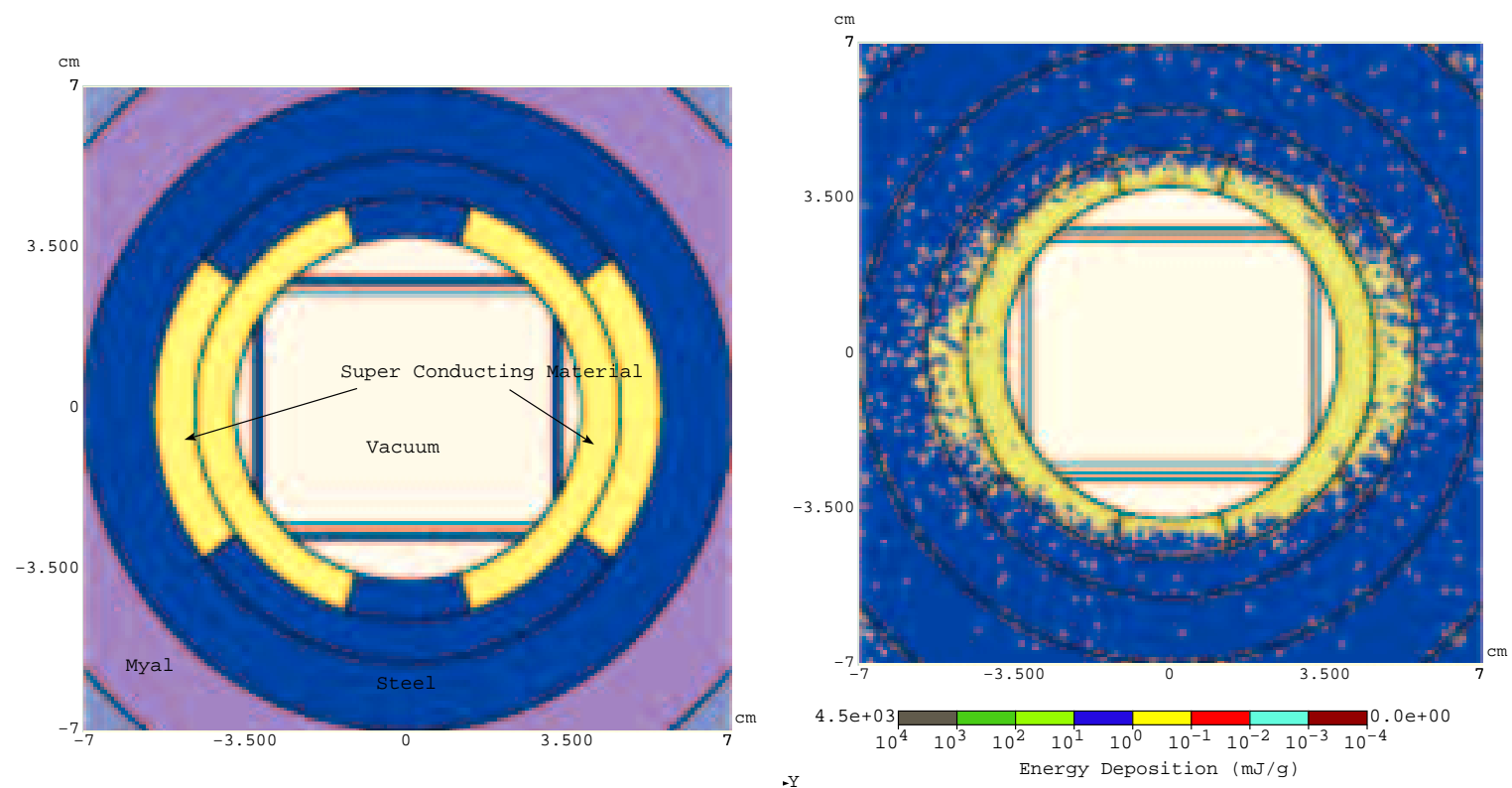

Figure 2: A cross-sectional view of the SC dipole in the MARS model (left) and energy deposition isocontours at shower maximum in the first dipole (right).
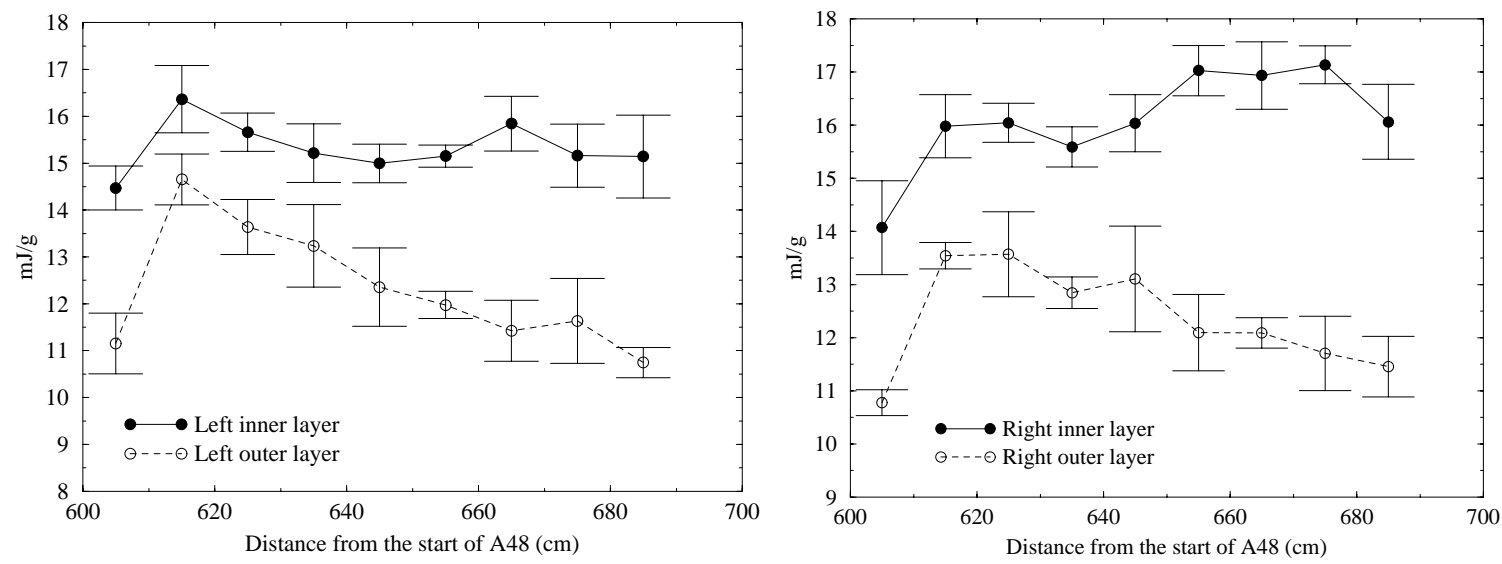

Figure 3: Energy deposition in the inner and outer SC coils of the first dipole. 


\section{Increasing Protection Efficiency}

Most of the energy deposited in the dipole's SC coils is due to electromagnetic showers induced by photons from $\pi^{0}$-decays, caused by high-energy proton interactions in the collimator. The primary goal of the collimator - CDF protection - is nicely achieved with its optimized parameters $(0.5 \mathrm{~m}$ of steel and configuration). The CDF detector sits at about 50 meters downstream with the $\mathrm{B} \varnothing$ dipoles and quadrupoles serving as a perfect active shielding against low-energy secondaries generated at the A48 location. From the other hand, a $0.5 \mathrm{~m}$ collimator (about three nuclear inelastic interaction lengths $\lambda$ in steel) is too short to absorb the full cascade induced by a $1-\mathrm{TeV}$ proton, resulting in an excessive irradiation of the dipoles at an AKP.

There is no room for a significant increase of the collimator length. Four possible cases have been expressly studied to reduce energy deposition in the dipole downstream of the A48 collimator:

1. The collimator length is increased by one interaction length $(17 \mathrm{~cm}$ of steel), i.e., from 0.5 to $0.67 \mathrm{~m}$.

2. In addition, a $0.34 \mathrm{~m}(2 \lambda)$ steel mask with a round $2.5-\mathrm{cm}$ radius aperture is placed immediately upstream of the first dipole.

3. Same as (2) with tungsten used instead of steel.

4. Same as (3) with first $0.3 \mathrm{~m}$ of the $0.67-\mathrm{m}$ collimator made of tungsten.

Fig. 4 (left) shows that energy deposition is down by about $22 \%$ for Case 1. Using a steel mask additionally (Case 2) doubles the protection efficiency, reducing the peak energy deposition by $44 \%$. It is further reduced in Case 3, but the maximum reduction is achieved in Case 4 (Fig. 4 (right)): the peak energy deposition in the inner coil is about $3.5 \mathrm{~mJ} / \mathrm{g}$ - almost a factor of five reduction compared to the baseline case. Unfortunately, even this unlikely configuration (higher cost, lack of room) of the A48 collimator and additional mask is not sufficient to prevent a quench of the first dipole.
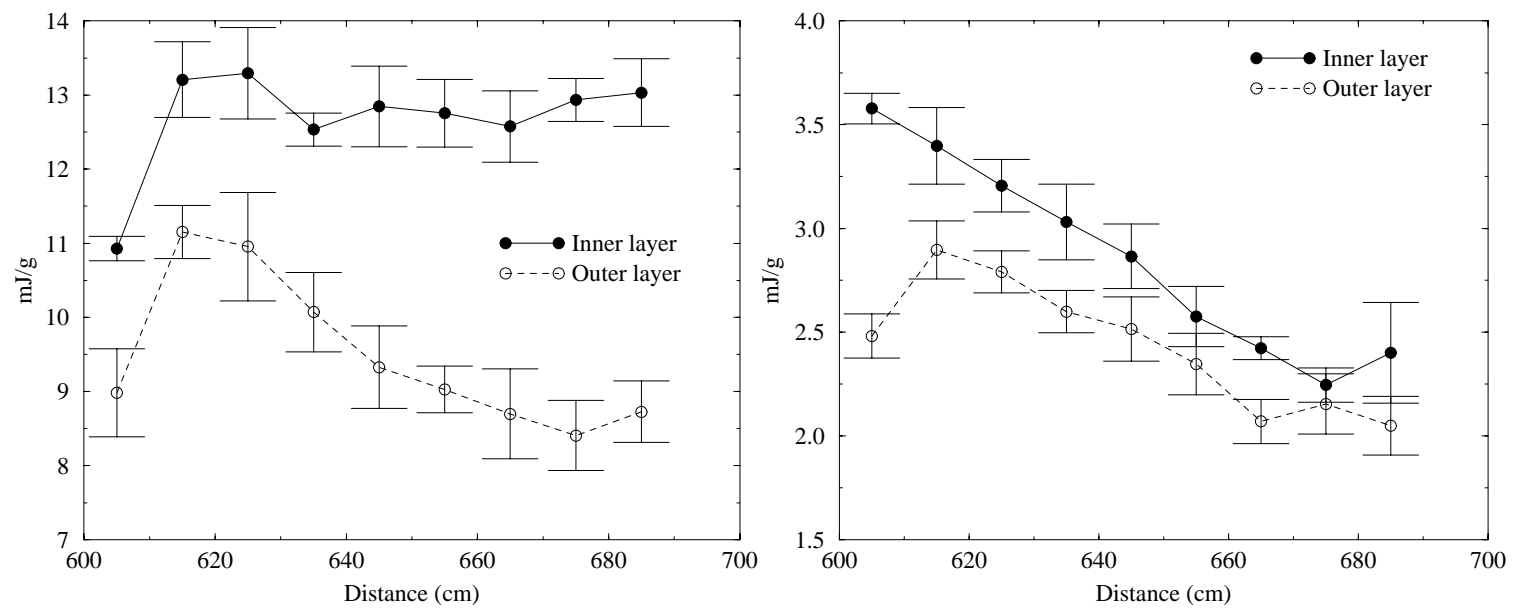

Figure 4: Energy deposition in the horizontal plane of the inner and outer SC coils along the first dipole in Case 1 (left) and Case 4 (right). 


\section{Conclusion}

The new A48 collimator will reliably protect the CDF detector at an abort kicker prefire, but it is not capable to eliminate a quench of the first SC dipole downstream in such an event. If such an incident occurs, it will probably result in a quench with a peak energy deposition in the coil about 30 times above the quench limit. Possible modifications in the A48 region considered in this note would reduce this factor up to five times, but it is likely that a quench in the first dipole would still occur. The abort kicker prefires are however fairly scarce and even a simple A48 collimator to be installed this summer represents actually a practical way to reduce radiation loads to the dipole in such an event.

\section{Acknowledgments}

We are thankful to Sasha Drozhdin for generating a file of AKP protons at A48.

\section{References}

[1] A.I. Drozhdin et al., "Tevatron Abort Kicker Prefire Simulations", Beams-doc-648-v1, June 2003.

[2] N.V. Mokhov, A.I. Drozhdin, "A48 Collimator: Beam Physics Justification and Simulation", Beams-doc-646-v1, June 2003.

[3] A.I. Drozhdin et al., "Beam Loss and Backgrounds in the CDF and DØ Detectors due to Nuclear Elastic Beam-Gas Scattering”, Fermilab-FN-734 (2003).

[4] L. Y. Nicolas, A. I. Drozhdin, N. V. Mokhov, "MARS Simulation of Beam Loss and New Collimator Impact on the CDF and DØ Subdetectors", DØ-Note-4129(2003), CDF-Note6407(2003).

[5] I.S. Baishev, A.I. Drozhdin, N.V. Mokhov, "STRUCT Program User's Reference Manual”, SSCL-MAN-0034 (1994), http://www-ap.fnal.gov/ drozhdin/

[6] N.V. Mokhov, "The MARS Code System User's Guide”, Fermilab-FN-628 (1995); N.V. Mokhov, O.E. Krivosheev, "MARS Code Status", Proc. Monte Carlo 2000 Conf., p. 943, Lisbon, October 23-26, 2000, Fermilab-Conf-00/181 (2000); N.V. Mokhov, "Status of MARS Code", Fermilab-Conf-03/053 (2003); http://www-ap.fnal.gov/MARS/. 